\title{
Pediatric Liver Transplantation as Enzyme Replacement Therapy for Rare Metabolic Diseases with No Structural Liver Damage
}

\author{
Neslihan Celik', [MD] \\ ORCID: 0000-0003-3493-471X
}

Remzi Emiroğlu', [MD]

ORCID: 0000-0002-9905-3182
'Department of Organ Transplantation, Acibadem University Atakent Hospital, Istanbul, Turkey.

This study has not been presented previously in a congress or symposium.

Corresponding Author: Neslihan Celik Department of Organ Transplantation, Acibadem University Atakent Hospital, Istanbul, Turkey. E-mail: nescelik60@gmail.com

https://doi.org/10.32552/2021.ActaMedica.672

\section{ree) ABSTRACT Cere}

Objective: Very rare monogenic metabolic diseases without structural liver damage can be cured by liver transplantation. This process is a surgical enzyme replacement therapy, and defective enzymes may or may not be confined to the liver. The aims of this single center study of children with metabolic diseases showing structurally normal liver parenchyma were to analyze the indications and post-operative outcomes of liver transplantation, identification of developmental and metabolic benefits of the procedure with recognition of peri-operative difficulties to improve the success rate.

Materials and Methods: Patients under the age of 19-year-old who underwent liver transplantation for metabolic disorders with no structural liver injury between January 2015 and June 2021 analyzed retrospectively. Patient and graft survivals, indications for transplantation, presence of extra-hepatic enzyme deficiency causing other organ damage, inclusion of simultaneous or sequential kidney transplantation, immunosuppressive protocols, post-transplant complications, and metabolic outcomes were identified.

Results: Eight children with primary hyperoxaluria type $1(n=4)$, Maple syrup urine disease $(n=1)$, Crigler-Najjar syndrome type $1(n=1)$, familial hypercholesterolemia $(n=1)$ and propionic acidemia $(n=1)$ received left lobe $(n=6)$ and left lateral segment (2) allografts from living donors. The median age of 4 girls and 4 boys at time of transplantation was 6.8 years (range 2.2-12.7 years). The median follow-up time was 3.3 years (range 1.5-5.7 years). The most common post-transplant complications were biliary system complications and infections and, two patients died because of sepsis. Six patients are alive with normal functioning allografts and metabolically stable on unrestricted diet.

Conclusion: Liver transplantation is a lifesaving treatment and improves patient's and parent's life quality for metabolic disorders with no parenchymal injury despite strict dietary restrictions and medical therapies. Especially, living donor liver transplantation is very important for populations with very low organ donation rates.

Keywords: Living donor liver transplantation, children, metabolic disease 


\section{INTRODUCTION}

Very rare monogenic metabolic diseases without structural liver damage can be cured by liver transplantation (LT). This process is a surgical enzyme replacement therapy, and the defective enzymes may or may not be confined to the liver (Table-1). Transplantation decision is simple for metabolic disorders with liver injury leading liver failure, but systemic consequences of deficient enzymes are the main reasons for LT when there is no liver distortion in histological evaluation. Transplantation might be a lifesaving or an organ saving procedure and/or an option for better quality of life due to severe systemic manifestations in this particular group of patients [1-9].

The aims of this single center study of children with metabolic diseases presenting structurally normal liver parenchyma were to analyze the indications, appropriate timing and post-operative outcomes of liver transplantation, identification of developmental and metabolic benefits of the procedure along with recognition of peri-operative difficulties to improve the success rate.

\section{MATERIALS and METHODS}

Between January 2015 and June 2021, patients under the age of 19-year-old who underwent liver transplantation at Acibadem University Hospital with diagnosis of metabolic disorders without structural liver damage were analyzed retrospectively. Operative techniques, patient and graft survivals, post-transplant surgical and medical complications, immunosuppressive protocols, and metabolic status of patients were identified. Donor and recipient characteristics were documented at time of transplantation and descriptive statistics were defined as median with minimum and maximum values. Patient and graft survivals were given according to primary diagnoses of the patients.

This study was approved by institutional review board with 2021/11-21 protocol number.

\section{RESULTS}

Between January 2015 and June 2021, eight children with five different hereditary metabolic disorders with no liver injury received liver allografts via living donor liver transplantation (LDLT). These included primary hyperoxaluria type 1 (PHO1, $n=4)$, Maple syrup urine disease (MSUD, $n=1$ ), Crigler-Najjar syndrome type 1 (CN1, $\mathrm{n}=1$ ), familial hypercholesterolemia ( $F H C, n=1$ ) and propionic acidemia $(P A, n=1)$. Six patients received left lobes (LL) and 2 were transplanted with left lateral segments (LLS) from living related donors. The median age of 4 boys and 4 girls at time of transplantation was 6.8 years (range 2.212.7 years). The body weight distribution of the patients was $18.7 \mathrm{~kg}$ median weight with range of $9.4-36 \mathrm{~kg}$. The median follow-up time was 3.3 years (range 1.5-5.7 years) after LDLT. Two patients with PHO1 died because of pneumonia related sepsis and urinary sepsis at 0.15 and 3.9 years respectively after their transplantations. Other 6 patients are alive with normal liver function on mono or dual immunosuppressive therapies. All patients are metabolically stable on unrestricted diet. There

Table 1. Classification of liver-based metabolic disorders with normal liver parenchyma [1-9], $\left({ }^{*}\right.$ Argininosuccinic aciduria).

\begin{tabular}{|l|l|}
\hline Metabolic disease with no structural liver injury & Extra-hepatic involvement of deficient enzyme \\
\hline Deficient enzyme confined to the liver & Maple syrup urine disease \\
\hline Crigler-Najjar syndrome type 1 & Methyl malonic acidemia \\
Primary hyperoxaluria type 1 & Propionic acidemia \\
Urea cycle disorders (except ASA*) & \\
Familial hypercholesterolemia & \\
Hemophilia & \\
Factor VII deficiency & \\
Protein C and S deficiencies & \\
Factor H deficiency & \\
Amyloidosis type 1 & \\
\hline
\end{tabular}


was no portal vein or hepatic artery thrombosis, and no re-transplantation was performed. Bile leak in 3 patients was resolved after drainage $(n=1)$, and revision of biliary reconstruction $(n=2)$. Biliary stricture following bile leak was resolved by percutaneous transhepatic cholangiography (PTC) with balloon dilatation in one patient. Patient and donor characteristics, technique details and postoperative period are summarized in Table-2 and 3.

\section{DISCUSSION}

Monogenic metabolic disorders are the second most common disease group for pediatric liver transplantation after biliary atresia. LT rate for metabolic disorders increased from $10 \%$ to $16 \%$ in last decade and still comprises $12.6 \%$ of the pediatric waitlist. Five-year patient and graft survival rates were given as $88-95 \%$ in OPTN/SRTR data for metabolic diseases with deceased donor liver transplantation and more than $70 \%$ varying with the primary diagnosis according to LDLT reports [1-3, 10-12]. Inherited metabolic diseases with normal liver parenchyma may benefit from LT if they cause multisystemic consequences due to deficient enzymes. Indications and outcomes for LT differ according to extra-hepatic manifestations.

Primary hyperoxaluria type 1 is an autosomal recessive metabolic disorder caused by alanine glyoxylate aminotransferase (AGT) enzyme deficiency. AGT is a liver peroxisomal enzyme and deficiency causes increased oxalate production and urinary excretion. Oxalate accumulates in bone, skin, arteries, heart, peripheral nerves and retina causing systemic oxalosis. Calcium oxalate crystals are deposited in kidneys and impairs renal function. Pyridoxine, hyperhydration, citrate, neutral phosphate and diuretics might be used to delay the progression of renal disease. Unfortunately, peritoneal and hemodialysis are not effective in removal of oxalate but may slow down systemic oxalosis prior to the transplantation. $\mathrm{PHO} 1$ can be cured by LT after total native hepatectomy. Isolated kidney transplantation is not recommended since disease recurs because of enzyme deficient native liver. Partial native hepatectomy and auxiliary LT is contraindicated in PHO1 due to enzyme deficient residual native liver. Blood and urinary oxalate levels are normalized after LT if there is no systemic oxalosis with normal renal function. Preemptive isolated LT may be considered when GFR is between $40-60 \mathrm{ml} / \mathrm{min} / 1.73 \mathrm{~m} 2$, but combined liver and kidney transplantation is strongly recommended if GFR falls below $40 \mathrm{ml} /$ $\mathrm{min} / 1.73 \mathrm{~m} 2$. However, the mobilization and urinary excretion of the oxalate load lasts months to years in case of systemic oxalosis. Simultaneous liver and kidney transplantation may cause impairment of renal graft with existing systemic oxalosis load despite immunological benefits of the procedure. Sequential liver and kidney transplantation might be considered to reduce the systemic oxalate

Table 2. Patient characteristics (*age at transplantation, years, tmale, ‡female, $\S$ age at diagnosis, years).

\begin{tabular}{|c|c|c|c|c|c|c|}
\hline Case number & Primary diagnosis & AAT* $^{*}$ & Gender & $\mathrm{AAD}_{\S}$ & Comorbidities & Pre-transplant therapies \\
\hline 1 & PHO1 & 6.3 & $\mathrm{M} \dagger$ & 4 & $\begin{array}{l}\text { Nephrocalcinosis, } \\
\text { Severe systemic oxalosis, } \\
\text { Failure to thrive }\end{array}$ & Pyridoxine, hemodialysis \\
\hline 2 & PHO1 & 6.7 & M & 4 & $\begin{array}{l}\text { Urolithiasis, } \\
\text { Nephrocalcinosis, } \\
\text { Severe systemic oxalosis }\end{array}$ & Pyridoxine, hemodialysis \\
\hline 3 & PHO1 & 10.3 & M & 2 & Urolithiasis & Pyridoxine, hemodialysis \\
\hline 4 & PHO1 & 10.4 & $F \neq$ & 5 & $\begin{array}{l}\text { Urolithiasis, } \\
\text { Nephrocalcinosis, } \\
\text { Severe systemic oxalosis }\end{array}$ & Pyridoxine, hemodialysis \\
\hline 5 & MSUD & 6.9 & $F$ & At birth & Poor neurocognitive function & $\begin{array}{l}\text { Protein restricted diet } \\
\text { (noncompliant) }\end{array}$ \\
\hline 6 & CN1 & 12.7 & $F$ & 8 & Poor school performance & No therapy \\
\hline 7 & $\mathrm{FHC}$ & 4.4 & $F$ & 4 & $\begin{array}{l}\text { Aortic valve calcification and } \\
\text { mild insufficiency }\end{array}$ & $\begin{array}{l}\text { Statins, omega-3, } \\
\text { coenzyme-Q }\end{array}$ \\
\hline 8 & PA & 2.2 & M & At birth & - & Protein restricted diet \\
\hline
\end{tabular}




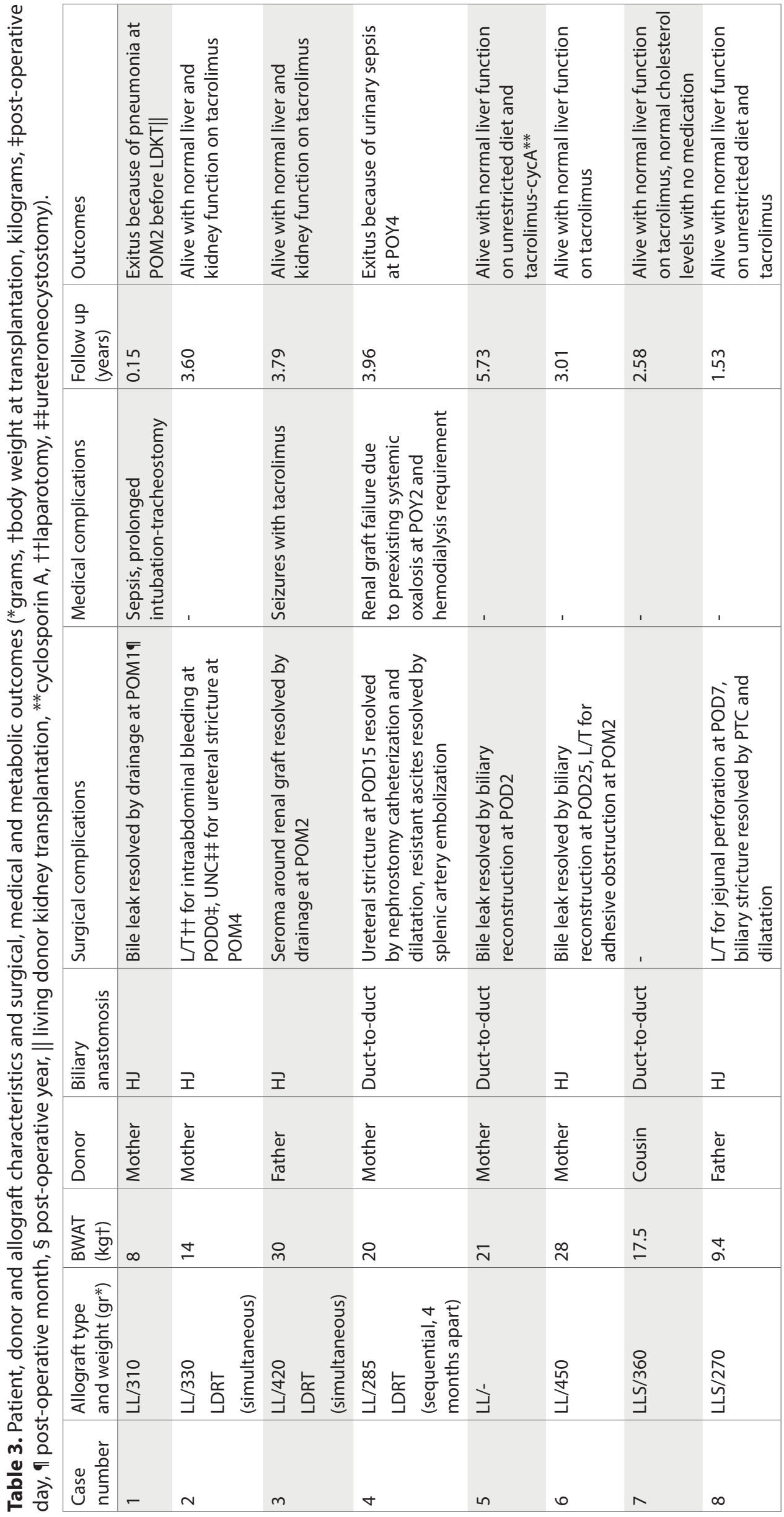


load by hemodialysis after LT, but dialysis is not an effective way of oxalate removal by any means $[1,13-19]$. Native nephrectomy may also help decreasing amount of mobilized oxalate and urinary stone complications in case of combined transplantation. LDLT and LDKT especially from same donor either simultaneous or sequential are favorable in terms of immunological point of view but needs to be assessed very carefully about risks for the donor. Previously we reported our experience with 4 pediatric and one adult $\mathrm{PHO} 1$ patients [20]. We lost two of four pediatric patients at post-transplant 2 nd month and 4th year due to severe pneumonia and urinary sepsis. Patient with urinary sepsis had renal allograft failure at second year due to preexisting systemic oxalosis mobilization, excretion and accumulation at renal graft.

Maple syrup urine disease (MSUD) is an autosomal recessive inherited disorder with defect in the branched-chain alpha ketoacid dehydrogenase $(B C K D H)$ complex. Valine, leucine, and isoleucine amino acids cannot be fully metabolized, and toxic metabolites cause metabolic decompensation despite aggressive therapy with dietary restriction of affected amino acids and dietary supplements. Neurocognitive function continues to decline any time when metabolic crises and cerebral edema occur during episodes of common infections and injuries. Liver transplantation is demonstrated to be effective in neurodevelopmental status of classical/severe MSUD besides improving children's and families' quality of life due to severe protein restriction and frequent hospitalizations. LT is crucial especially for patients with poor access to health centers with metabolic disease experts. LT cures the disease but cannot reverse existing spasticity, dystonia, or mental retardation. It is important to inform families of patients with poor metabolic control about LT option before irreversible neurological complications occur. Mazariegos et al reported 100\% patient and graft survival in their 35 -patient series at $4.5 \pm 2.2$ years of follow-up period. MSUD patients with normal liver function tests are also good candidates for domino liver transplantation [2,3,8,21-23]. We performed LDLT for one patient with already declined neurocognitive function since patient was not compliant with her diet and experienced multiple episodes of metabolic crises. She underwent LT at age of 6.9 years. She is metabolically stable on unrestricted diet and no further neurocognitive deterioration was reported during her posttransplant follow up.

Propionic academia is an autosomal recessive inherited metabolic disease with defective propionyl-CoA carboxylase enzyme. Although there is partial correction of metabolic defect, liver transplantation is indicated in case of frequent metabolic crises despite optimum medical therapy to improve the quality of life and limit the neurocognitive impairment and/or cardiomyopathy. Barshes et al reported $72 \%$ patient survival in their review after LT on unrestricted diet. Generally, organic acidemia patients are allowed to have approximately 0.8 grams protein $/ \mathrm{kg}$ body weight protein-limited diet or specially designed enteral formulas [1,24-26]. We transplanted one patient with PA who had very early diagnosis and started strict diet with medical therapy. Unfortunately, patient experienced multiple hyperammonemia episodes and hospitalizations at times of simple infections and injuries. Patient received LDLT at age of 2.2 and had no metabolic crises since then on unrestricted diet.

Crigler-Najjar syndrome type 1 is a rare autosomal recessive inherited metabolic disorder with severe indirect hyperbilirubinemia. Total deficiency of uridyl-diphosphate glucuronosyl transferase enzyme (UGT1A1) activity causes accumulation of unconjugated bilirubin leading severe encephalopathy. Patients require phototherapy to decrease bilirubin levels and prevent irreversible kernicterus. Despite prolonged phototherapy (12 to 20 hours/day), the children remain jaundiced, and neurologic damage may occur at any time and more than $20 \%$ of young adults develop permanent neurological lesions under extensive phototherapy. Thirty percent hepatic UGT1A1 activity is required to preserve normal bilirubin homeostasis in total body and LT is recommended as a cure within the first year of life before the onset of neurological sequela besides improving patients' and families' quality of life. Strauss et al reported $100 \%$ patient and graft survival in their $16 \mathrm{CN} 1$ patient series with normal bilirubin levels $[1,27]$. We performed LDLT for one CN1 patient who was diagnosed very late and neurologically affected with poor school performance. Patient was treated as Gilbert syndrome until 8 years old and after that she was on no therapy. Her indirect bilirubin levels were 
around $17-18 \mathrm{mg} / \mathrm{dL}$ at time of transplantation and normalized in 12 days at post-operative follow up. Her school success has been improved drastically since LT with normal bilirubin levels.

Familial hypercholesterolemia is an autosomal dominant inherited disorder characterized by isolated elevation of plasma low-density lipoprotein (LDL) cholesterol. LDL receptor defects or anomalies of Apolipoprotein B cause high plasma LDL cholesterol levels and it is associated with high risk of very early cardiovascular problems. LT is indicated before advanced cardiovascular disease to minimize the operative risks and to avoid combined heart and liver transplantation. Near normal levels of LDL receptors and cholesterol can be achieved since normal liver contains $50-75 \%$ of LDL receptors but statin use might be required after the transplantation to maintain completely normal blood cholesterol levels. LDLT is a good option in regions with limited deceased donation but it might be hard to find a normolipidemic living related donor and parents are deemed as unsuitable candidates since they might be undiagnosed FHC heterozygotes in homozygous FHC cases. There are controversial statements in the literature but many successful LTs with normolipidemic heterozygote parental donors were reported with or without need of statin use in their post-transplantation follow up. Unfortunately, side effects of immunosuppressant therapies can also give rise to renal damage, heart failure, and bone marrow suppression and especially cyclosporine may cause hyperlipidemia but according to literature 10-year survival rate exceeds $70 \%[1,28-33]$. We performed LDLT for one homozygous FHC patient from her cousin. Patient's parents were heterozygotes for LDLRgene mutation with slightly high cholesterol levels. Patient was diagnosed at age of 4 years and was already affected with very high LDL cholesterol levels. Her cardiac work up showed aortic calcification and mild aortic insufficiency with intimal thickening in bilateral common carotid arteries. Patient's lipid profile was normalized and stayed normal with no statin use after LT.

As a conclusion, liver transplantation is a lifesaving treatment and improves patients' and parents' quality of life for children with metabolic disorders with no parenchymal injury despite dietary restrictions and several medical treatment modalities. Timing of the liver transplantation is crucial not to allow for neurocognitive and other organ impairment. Especially, LDLT is a good option for populations with very low organ donation rates enabling appropriate timing of the operation with extra immunological benefits.

\section{CONFLICT Of INTEREST}

No conflict of interest was declared by the authors.

\section{ree REFERENCES Cen}

[1] Darwish AA, McKiernan P, Chardot C. Paediatric liver transplantation for metabolic disorders. Part 1: Liverbased metabolic disorders without liver lesions. Clin Res Hepatol Gastroenterol 2011; 35(3): 194-203.

[2] Mazariegos G, Shneider B, Burton B, et al. Liver transplantation for pediatric metabolic disease. Mol Genet Metab 2014; 111(4): 418-27.

[3] Hansen K, Horslen S, Metabolic liver disease in children. Liver Transpl 2008; 14: 713-733.

[4] Kayler LK, Merion RM, Lee S et al, Long-term survival after liver transplantation in children with metabolic disorders. Pediatr Transplant 2002; 6: 295-300.

[5] Kayler LK, Rasmussen CS, Dykstra DM et al, Liver transplantation in children with metabolic disorders in the United States. Am J Transplant 2003; 3: 334-339.

[6] Stevenson T, Millan MT, Wayman $K$ et al. Long-term outcome following pediatric liver transplantation for metabolic disorders. Pediatr Transplant 2010; 14: 268-275.
[7] Sokal EM, Sokol R, Cormier V et al, Liver transplantation in mitochondrial respiratory chain disorders. Eur J Pediatr 158 (Suppl. 2) 1999: 81-84.

[8] McKiernan PJ, Ganoza A, Squires JE et al. Evolving Trends in Liver Transplant for Metabolic Liver Disease in the United States. Liver Transpl 2019; 25(6): 911-921.

[9] Hadžić N. Metabolic Indications for Pediatric Liver Transplantation: A Slow Train Coming. Liver Transpl 2019; 25(6): 829-830.

[10] Kwong A, Kim WR, Lake JR et al. OPTN/SRTR 2018 Annual Data Report: Liver. Am J Transplant 2020; 20 Suppl s1: 193299.

[11] Kasahara M, Sakamoto S, Fukuda A. Pediatric living-donor liver transplantation. Semin Pediatr Surg 2017; 26(4): 224232.

[12] Hong SK, Yi NJ, Yoon KC et al. Outcomes of Pediatric Liver Transplantation in Korea Using Two National Registries. J Clin Med 2020; 9(11): 3435. 
[13] Cochat P, Liutkus A, Fargue $S$, et al. Primary hyperoxaluria type 1: still challenging! Pediatr Nephrol 2006; 21 (8): 107581.

[14] Fargue S, Harambat J, Gagnadoux MF et al. Effect of conservative treatment on the renal outcome of children with primary hyperoxaluria type 1. Kidney Int 2009; 76(7): 767-73.

[15] Cibrik DM, Kaplan B, Arndorfer JA et al. Renal allograft survival in patients with oxalosis. Transplantation 2002; 74(5): 707-10.

[16] McDonald JC, Landreneau MD, Rohr MS et al. Reversal by liver transplantation of the complications of primary hyperoxaluria as well as the metabolic defect. $\mathrm{N}$ Engl J Med 1989; 321(16): 1100-3.

[17] Perera MT, McKiernan PJ, Sharif $K$ et al. Renal function recovery in children undergoing combined liver kidney transplants. Transplantation 2009; 87(10): 1584-9.

[18] Kemper MJ. The role of preemptive liver transplantation in primary hyperoxaluria type 1. Urol Res 2005; 33(5): 376-9.

[19] Malla I, Lysy PA, Godefroid N et al. Two-step transplantation for primary hyperoxaluria: cadaveric liver followed by living donor related kidney transplantation. Pediatr Transplant 2009; 13(6): 782-4.

[20] Ozer A, Aktas H, Bulum B et al. The experience of combined and sequential liver and kidney transplantation from a single living donor in patients with primary hyperoxaluria type 1. Pediatr Transplant 2019; 23(4): e13406.

[21] Mazariegos GV, Morton DH, Sindhi R et al. Liver transplantation for classical maple syrup urine disease: long-term follow-up in 37 patients and comparative United Network for Organ Sharing experience. J Pediatr 2012; 160(1): 116-121.

[22] Celik N, Kelly B, Soltys K et al. Technique and outcome of domino liver transplantation from patients with maple syrup urine disease: Expanding the donor pool for live donor liver transplantation. Clin Transplant 2019; 33(11): e13721.
[23] Celik N, Squires JE, Soltys $K$ et al. Domino liver transplantation for select metabolic disorders: Expanding the living donor pool. JIMD Rep 2019; 48(1): 83-89.

[24] Barshes NR, Vanatta JM, Patel AJ et al. Evaluation and management of patients with propionic acidemia undergoing liver transplantation: a comprehensive review. Pediatr Transplant 2006; 10(7): 773-81.

[25] Rela M, Battula N, Madanur M et al. Auxiliary liver transplantation for propionic acidemia: a 10-year followup. Am J Transplant 2007; 7(9): 2200-3.

[26] Fraser JL, Venditti PC. Methylmalonic and Propionic Acidemias: Clinical Management Update. Curr Opin Pediatr 2016; 28(6): 682-693.

[27] Strauss KA, Ahlfors CE, Soltys K et al. Crigler-Najjar Syndrome Type 1: Pathophysiology, Natural History, and Therapeutic Frontier. Hepatology 2020; 71(6): 1923-1939.

[28] Varret M, Abifadel M, Rabes JP et al. Genetic heterogeneity of autosomal dominant hypercholesterolemia. Clin Genet 2008; 73(1): 1-13.

[29] Bilheimer DW, Goldstein JL, Grundy SM et al. Liver transplantation to provide low-density-lipoprotein receptors and lower plasma cholesterol in a child with homozygous familial hypercholesterolemia. N Engl J Med 1984; 311(26): 1658-64.

[30] Sokal EM, Ulla L, Harvengt C et al. Liver transplantation for familial hypercholesterolemia before the onset of cardiovascular complications. Transplantation 1993; 55(2): 432-3.

[31] Revell SP, Noble-Jamieson G, Johnston $P$ et al. Liver transplantation for homozygous familial hypercholesterolaemia. Arch Dis Child 1995; 73(5): 456-8.

[32] East C, Grundy SM, Bilheimer DW. Normal cholesterol levels with lovastatin (mevinolin) therapy in a child with homozygous familial hypercholesterolemia following liver transplantation. JAMA 1986; 256(20): 2843-8.

[33] Ishigaki $Y$, Kawagishi N, Hasegawa $Y$ et al. Liver Transplantation for Homozygous Familial Hypercholesterolemia. J Atheroscler Thromb. 2019; 26(2): 121-127. 